\title{
A NARRATIVE EXPOSURE TREATMENT AS INTERVENTION IN A REFUGEE CAMP: A CASE REPORT
}

\author{
Frank Neuner $^{1}$ \\ Margarete Schauer ${ }^{1}$ \\ Walton T. Roth ${ }^{2}$ \\ Thomas Elbert ${ }^{1}$
}

${ }^{1}$ University of Konstanz

${ }^{2}$ Stanford University and Palo Alto Veterans Affairs Medical Center

Adress correspondence to: Frank Neuner, Department of Psychology, Fach D25, University of Konstanz, D-78457 Konstanz, Germany, e-mail: frank.neuner@uni-konstanz.de 


\begin{abstract}
In a severely traumatised Kosovar refugee living in a Macedonian refugee camp during the Balkan War, we applied a Narrative Exposure Treatment (NET). NET is a pragmatic shortterm approach that integrates effective therapeutic components deriving from Cognitive Behaviour Therapy and Testimony Therapy. Outcome was evaluated by clinical examination and the Posttraumatic Stress Diagnostic Scale (PDS). Three sessions of NET were enough to afford considerable relief, although some PTSD symptoms remained. Our experience indicates that Narrative Exposure is a promising and realistic approach for the treatment of even severely traumatised refugees living in camps. In addition, it can provide valid testimonies about human rights violations without humiliating the witness.
\end{abstract}

\title{
Key words
}

Narrative Exposure Therapy, refugee camp, PTSD, Kosovo war, emergency psychological trauma aid 
Many refugees who flee from dictatorships or war-torn areas report having witnessed or experienced atrocities, torture and other extremely stressful events. A considerable proportion of refugees (up to 35\%) suffers from Posttraumatic Stress Disorder (PTSD) as a consequence of these traumatic events (e.g. Mollica et al. 1993). The need for psychosocial interventions for the assistance of traumatized refugees is enormous.

In recent years research on therapy of PTSD has made a considerable progress. Cognitive behavioural exposure techniques have proven to be the most successful interventions in treating PTSD (Foa \& Meadows, 1997). In CBT, exposure to the traumatic memory is achieved by asking the client to repeatedly report the traumatic event in detail. Clients are encouraged to express their emotions during recall. The treatment is thought to take effect through the habituation of the emotional response that is conditioned to reminders of the traumatic event.

However, exposure techniques applied to clients suffering from traumatic stress after rape or motor vehicle accidents cannot be easily transferred to refugees who flee from wars and dictatorships. One problem is that in CBT, exposure to the traumatic stimuli is usually obtained through the processing of the memory of the worst event the client remembers. This is difficult to achieve with survivors of organised violence as most of them do not experience single traumatic events, but a series of extremely stressful experiences during their persecution (e.g. Mollica et al., 1993). The length of time these survivors had been exposed to violence can span several years, and often the most stressful event among the sequence of traumas is impossible to identify.

One specialised exposure-oriented treatment approach can overcome this difficulty. Testimony Therapy (TT; Cienfuegos \& Monelli 1983; Weine, Kulenovic, Pavkovic, \& Gibbons, 1998), is a short-term treatment method that was especially developed for survivors of torture 
and other severe human rights violations. TT aims at the construction of a detailed and coherent report of the survivor's biography including an explicit description of the traumatic events. The written testimony created by the patient in cooperation with a therapist is used for documentary and political purposes.

The procedures of cognitive behavioural exposure therapy and TT share many common features. As in CBT, prolonged exposure to the traumatic material is realised through reporting about it. This may promote the habituation of emotional and physiological reactions to reminders of the traumatic events and reduce symptoms in this way. But the focus of TT is not on habituation but on the reconstruction of the shattered autobiographic memories of traumatic experiences. The importance of the structure of autobiographic memories is also stressed in new cognitive theories of PTSD (e.g. Ehlers \& Clark, 2000). These models try to identify factors relevant for the efficacy of treatment beyond habituation. One assumption is that, compared to memories of normal events, autobiographical memories of traumatic events are more fragmented and incomplete. The reorganisation of these memories towards a coherent narration (one aim of TT) is believed to promote the cognitive and emotional processing of the trauma.

The Narrative Exposure Treatment (NET) applied in this study is a standardised and manualised variant of Testimony Therapy. It includes several elements that have been adapted from CBT. Like CBT, NET facilitates both the reconstruction of autobiographic memory and habituation. Thus the therapist must be aware of both processes in treatment. In addition, NET includes an educational component, in which the cognitive-behavioural oriented theory behind the disorder and the procedure is explained to the client.

The NET procedure can be outlined as follows: Treatment starts with a detailed assessment and explanation of the treatment rationale. In the following sessions the client constructs a 
consistent narration of his biography in cooperation with the therapist. The focus of the therapy lies in completion and integration of the initial fragments about the traumatic events into a whole which encompasses the sensory, emotional and cognitive experiences of the incident. The therapist helps clarify ambiguous descriptions. Inconsistencies in the client's report are gently pointed out. The therapist may ask about recurring body sensations or thoughts. The client is encouraged to tell the traumatic events in as much detail as possible and to reveal the emotions and perceptions experienced at that moment. A translator, oriented beforehand to the psychological goals, may be necessary.

The therapist writes down the client's narration (or the translation if necessary). In the subsequent session, the report that was constructed in the previous session is read to the client (simultaneously translated by the interpreter if necessary) and s/he is asked to correct it and to add further details, especially about the emotional and cognitve appraisal of the events reported. The procedure is repeated across sessions until a final version of the client's biography is reached. Three to four sessions, spanning up to two hours each, are usually necessary for treatment.

In the last session the complete document is read and the client, the translator and the therapist sign the written narration. One copy of the signed document is handed to the client; another is kept for therapeutic documentary purposes. With the agreement of the client, another copy is passed on to human rights organisations for documentary and advocacy purposes or published in another way.

\section{The case of Aferdita}

Narrative Exposure Treatment was tested in a refugee camp in Macedonia in cooperation with an international humanitarian aid organisation. Key objectives of the psychosocial programme during the Kosovo crisis included identification of psychologically disturbed 
refugees, first aid and short-term treatment. Emphasis was put on mobilising the client's social support network. Narrative Exposure was implemented only when other methods of stabilising the client's personal or social situation had failed.

Aferdita (the name is fictitious), a 24-year old student from Pristina, was carried into the medical health tent on a stretcher seven days after she had arrived in the camp. After regaining consciousness, she reported that she had had repeated episodes of loss of consciousness for several years. As the physicians could not find any reason for these episodes, she was referred to the mental health unit.

A translator was necessary for assessment and for treatment. An interview by a mental health professional revealed that Aferdita suffered from diverse dissociative symptoms, such as emotional numbness and out-of-body experiences. Posttraumatic Stress Disorder (according to DSM-IV criteria) was present as she experienced marked intrusion, avoidance and hyperarousal symptoms. She was severely depressed and kept social contact with no one but her cousin (the only other member of the family in the camp). The Posttraumatic Diagnostic Scale (PDS; Foa 1995) revealed maximum scores for each symptom except partial amnesia. Aferdita reported that the symptoms had persisted for five years.

Efforts to alleviate her suffering through medication, counselling and problem-solving had failed. We suggested applying Narrative Exposure. The limits of scientific knowledge about its effectiveness under the given circumstances were made fully clear to her. Aferdita agreed, since she wanted the truth about her persecution to be known.

The first session of NET revealed a long history of persecution of Aferdita and her family, which included many potentially traumatic events. She could report her past without difficulty until she came to the following scene, the recounting of which visibly affected her. 
„I was the first to see the paramilitaries forcefully entering our house. This happened six years ago. I fainted in that moment. Later I was told that they searched the house. When I came to consciousness, I was lying on my back on the ground in the backyard. A man in uniform stood on top of me pointing a gun to my forehead and screaming at my father: 'Give us weapons or money or you will lose your daughter!'"

After Aferdita reported this event the first time, she fainted. Some minutes later, after regaining consciousness, she expressed the desire to continue therapy and was intent on telling the whole story without fainting. With sustained encouragement and support of the therapist, she managed to reconstruct the complete episode. Within three sessions (about 70 min each) she had created a nearly complete narration of her autobiography including several traumatic events. Contrary to her own expectation, therapy was no longer interrupted by fainting spells. After the third Narrative Exposure session, which took place within five days, Aferdita had no more losses of consciousness. Her mood improved dramatically. In view of the great number of refugees seeking help, her therapy was terminated at this point.

A follow-up examination one week later (just before her evacuation to the USA) revealed that Aferdita still suffered from mild PTSD symptoms including intrusions such as incidental nightmares and flashbacks. However, PDS-assessed Symptom Severity had decreased from 15 to 8 for Reexperiencing, from 15 to 9 for Arousal and from 18 to 8 for Avoidance. In particular, her marked dissociative symptoms had disappeared. She showed no signs of depression and started to relate to peers (for the first time in five years) at an activity site that was run by an international NGO. Aferdita phoned unexpectedly from her overseas exile about one year after the treatment. She expressed satisfaction with the treatment and reported that she was in "very good" condition because symptoms had fully remitted. 


\section{Discussion}

NET uses a combination of a testimony-like approach developed specially for survivors of severe human rights violations, together with elements of CBT. Our experiences with NET are promising. The explicit political aim of the approach clearly raised her motivation to report her traumatic events. She was able to substantially profit from three sessions of NET, although she initially suffered from severe symptoms and substantial restrictions in social functioning. NET was superior to psychoactive medication, counselling and problem-solving treatment and no negative consequences of NET have been observed in this case.

Of course, complete restoration of all areas of impaired functioning is hardly to be expected, particularly under the pressing circumstances in a refugee camp. In this case, a mild form of PTSD was still present right after treatment. But to our surprise NET proved to be particularly effective for the symptoms of dissociation and hyperarousal, the reduction or elimination of which enabled the patient to function again. It is uncertain if longer treatment would have brought about a more complete relief of symptoms. In further sessions clients would have been encouraged to go through their traumatic memories repeatedly, hopefully facilitating more habituation of emotional responses. However, given the limitations of the refugee camp setting, complete remission was not a practical goal, so we did not determine how much improvement is possible when this approach is extended.

One year after therapy, the patient reported that her symptoms had further improved after treatment. This positive outcome was probably affected by the fact that she had been removed to a safe exile after therapy. Further research is indicated to test the efficacy of NET for a wide group of refugees. In particular it is important to examine the long-term effect of NET for those less privileged survivors who have to stay in refugee settlements for many years or are forced to return to their country of origin. Furthermore, to control for 
sponaneous remission and unspecific treatment effects, a randomized controlled treatment study is necessary.

\section{Acknowledgements}

Research was supported by the Deutsche Forschungsgemeinschaft. 


\section{References}

CIENfuegOS, J., \& MONELLI, C. (1983). The testimony of political repression as a therapeutic instrument. American Journal of Orthopsychiatry, 53, 43-51.

FOA, E. B. (1995). Posttraumatic Stress Diagnostic Scale. National Computer Systems, Minneapolis, USA

FOA, E. B., \& MEADOWS, E. A. (1997). Psychosocial treatments for posttraumatic stress disorder. Annual Review of Psychology, 48, 449-480.

MOlliCA, R. F., DONElAN, K., TOR, S., LAVElle, J., EliAS, C., FRANKEL, M., \& BlendON, R. J. (1993). The effect of trauma and confinement on functional health and mental health status of Cambodians living in Thailand-Cambodia border camps. JAMA, 270, 581-586.

WEINE, S. M., KUlENOVIC, A. D., PAVKOVIC, I., \& GIBBONS, R. (1998). Testimony psychotherapy in Bosnian refugees: A pilot study. American Journal of Psychiatry, 155, 1720-1726.

EHLERS, A. \& CLARK, D. M. (2000). A cognitive model of posttraumatic stress disorder. Behavioural Research and Therapy, 38, 319-345 